\title{
Correlation between severity of diabetes mellitus, polyneuropathy and
}

\section{nasal mucociliary clearance}

\author{
Mirac Aysen Unsal $^{1 *}$, Suphi Bulgurcu ${ }^{2}$
}

\begin{abstract}
Objective: To examine the relationship between the severity of polyneuropathy and nasal mucociliary clearance times in patients with polyneuropathy and investigate how the presence and severity of electrophysiological polyneuropathy might affect mucociliary clearance in patients with diabetes mellitus.

Material and Methods: This prospective cross-sectional study was carried out in the Neurol-ogy and Ear, Nose and Throat (ENT) clinics in a tertiary hospital. The study included three groups of patients with 20 participants in each group (Group 1, patients with diabetic poly-neuropathy; Group 2, patients with non-diabetic polyneuropathy; and Group 3 , diabetes melli-tus patients with a normal nerve conduction study) Nasal saccharin test was performed on all patients.

Results: There was a statistically significant difference in the duration of nasal mucociliary clearance among the groups $(p=0.001)$. There was a positive, statistically significant $(p=0.007)$ correlation between the nasal mucociliary clearance duration and the severity of poly-neuropathy. The nasal mucociliary clearance duration increased with the severity of polyneu-ropathy.
\end{abstract}

Conclusion: Patients with diabetes mellitus are a special group, and preventable problems should be taken into consideration when examining nasal pathologies. It should be kept in mind that nasal mucociliary clearance dysfunction can be both a cause and a result in man-agement of diabetes mellitus patients, and thus it should be evaluated carefully.

Keywords: nasal mucociliary clearance, saccharin test, diabetes mellitus, diabetic polyneu-ropathy

\section{Introduction}

Effective ciliary activity and regularly regenerated airway surface liquids are required for the respiratory mucosal surfaces to remove accumulated foreign particles. Being essential for normal physiology, these two features are commonly referred to as mucociliary activity. The combined effect of the mucus and ciliary system is evaluated by mucociliary clearance meas-urement. And, mucociliary clearance (MC) can be determined by measuring the elimination time of inhaled aerosols (1)

Various environmental, metabolic and pharmacological factors have an effect on the mucocil-iary activity, including mucosal dryness, smoking, room temperature, hypoxia, hypercarbia, dehydration, $\mathrm{pH}$ changes, cystic fibrosis, primary ciliary dyskinesia, pharmacological agents such as phenylephrine, epinephrine, lidocaine, atropine, antihistamines, bacterial and viral infections, and allergic rhinitis. The abnormal ciliary function causes chronic upper and lower respiratory tract infections (2-4).
Assessment of mucociliary function started in the 1830 s, and migration of various substances placed on the nasal mucosa was examined by means of direct observation. Saccharin test is one of the methods used for this measurement (5-7). The circulation of the nasal airway is mediated by the autonomic nervous system. There are sympathetic and parasympathetic fibers of the autonomic system in the nose (8). Previous studies reported that nasal mucociliary clearance (NMC) in people with diabetes mellitus (DM) differed from the normal population due to dryness in the nasal membrane, increased nasal mucous viscosity, and abnormalities in small vessels $(9,10)$. We think that autonomic involvement of diabetic and nondiabetic polyneuropathies may also be a prominent factor in the disruption of the MC. The present study aimed to evaluate how NMC is affected by the presence of electrodiagnos-tic polyneuropathy in patients with DM, and compare the severity of diabetic and non-diabetic polyneuropathy to that of nasal mucociliary dysfunction. 


\section{Materials and Methods}

\section{Patients}

This prospective cross-sectional study was carried out in the Neurology and Ear, Nose, and Throat (ENT) clinics in a tertiary hospital. Before the study, an approval was obtained from the local Ethics Committee as well as written informed consent from each participant.

The study included three groups of patients, homogenous in terms of age and gender, with 20 participants in each group (Group 1, patients with Diabetic Polyneuropathy (DPN), DM pa-tients with abnormal nerve conduction study; Group 2, patients with non-diabetic polyneurop-athy (N-DPN); and Group 3, DM patients with a normal nerve conduction study)

A detailed history was taken from all patients. Data on duration of DM and the presence of peripheral neuropathy were collected. A detailed general physical examination and neurologi-cal examination were performed and documented by the same physicians. Nasal endoscopy was performed with a complete ENT examination and was evaluated in detail for nasal pa-thology. Patients who had upper respiratory tract infection, nasal surgery, septum deviation, concha hypertrophy, chronic otitis media, nasal discharge and nasal polyps and patients who were smoking and using pharmacological agents that would affect ciliary activity were ex-cluded. Patients with systemic diseases that might have affected ciliary activity were also excluded.

Saccharin test was done at least 3 hours after fasting and between 10-12 a.m. so that other factors such as coffee drinking, nutrition and circadian rhythm did not affect the test result.

\section{Saccharin test}

The saccharin test has been described by Andersen and modified by Rutland and Cole (6,7). MC measurement was performed for all patients and the control group by the saccharin test. The test was carried out at a room temperature with the patient seated and his/her head in the upright position. First of all, the patient was asked to clear the secretions in his/her nose. A1x1x1milimeter (1/4 saccharin tablet) size saccharin tablet was placed 1centimeter posterior from the anterior border of the inferior turbinate medial surface in one of the nasal cavities with the help of port cotton. The patient was asked not to sneeze, sniff, and bend his/her head forward. The patient was instructed to tell the moment when (s)he felt the taste, which was recorded as the NMC time.

Evaluation of the severity of polyneuropathy with electromyography (EMG)

All patients underwent nerve conduction study (NCS) using Medelec Synergy machine, Ox-ford, UK for measurement of sensory and motor latency, amplitude, and velocity. Motor con-duction studies consisted of stimulating the median nerve, ulnar nerve, common peroneal nerve and posterior tibial nerve while sensory conduction studies consisted of stimulating the median nerve, ulnar nerve and sural nerve.
In motor conductions, distal latency, conduction velocity, amplitude and $\mathrm{F}$ wave were evaluated. In sensory conduction, distal latency, conduction velocity and amplitude were evaluated. Patients with DPN and N-DPN in the first and second groups were divided into 3 groups according to the severity of their neuropathy. All NCSpositive patients were classified into following subgroups according to their outcomes: Mild-prolonged sensory latency and/or decreased velocity in sensory and motor nerves, with normal amplitude; moderate - above with mildly decrease sensory and/or motor amplitude; and severe - absent sensory potentials or severely reduced sensory and/or motor amplitudes.

\section{Statistical analysis}

A power analysis was performed before the study was designed. Twenty patients were en-rolled each in to three groups, with a total number of 60 participants. For statistical analysis, data were entered in Microsoft Excel format. Descriptive and analytic statistics were per-formed using the Statistical Package for the Social Sciences (SPSS) software for Windows, version 21.0 (SPSS Inc., Chicago, IL, USA). Independent t-test and One way-ANOVA test were applied to compare nominal data between the groups. The relationship between NMC duration and severity of polyneuropathy (both in DPN and N-DPN groups) was examined by Pearson's correlation analysis. A Pvalue $<0.05$ was regarded as statistically significant.

\section{Results}

Among the participants, the number of females and males was $12(60 \%)$ and $8(40 \%)$ in the first group, $11(55 \%)$ and $9(45 \%)$ in the second group, and $11(55 \%)$ and $9(10 \%)$ in the third group, respectively. The average age was $56.15 \pm$ $8,58.75 \pm 11.4$ and $57 \pm 13.3$ years in the first, second and third groups, respectively. The groups were statistically homogeneous in terms of gender and age $(\mathrm{p}>0.05)$

The average duration of NMC in the DPN, N-DPN and DM without polyneuropathy patient groups was $12.69 \pm 6.3$, $10.11 \pm 4$, and $6.76 \pm 1.4$ minutes, respectively. There was a statisti-cally significant difference in the duration of NMC among the groups $(\mathrm{p}=0.001)$ (Table 1).

Table1: Duration of NMC in each group of patients

\begin{tabular}{lcc} 
Patient groups & $\begin{array}{c}\text { Duration } \\
\text { of NMC } \\
\text { (minutes) }\end{array}$ & $\begin{array}{c}p \text { - } \\
\text { value }\end{array}$ \\
\hline $\begin{array}{l}\text { Patients with diabetic } \\
\text { polyneuropathy }\end{array}$ & $12.69 \pm 6.3$ & \\
$\begin{array}{l}\text { Patients with non-diabetic } \\
\text { polyneuropathy }\end{array}$ & $10.11 \pm 4$ & 0,001 \\
$\begin{array}{l}\text { DM patients without } \\
\text { polyneuropathy }\end{array}$ & $6.76 \pm 1.4$ & \\
\hline
\end{tabular}


There was a positive, statistically significant $(\mathrm{p}=0.007, \mathrm{r}=$ 0.42) correlation between the NMC duration and the severity of polyneuropathy. The NMC duration increased with the se-verity of polyneuropathy (Figure 1)

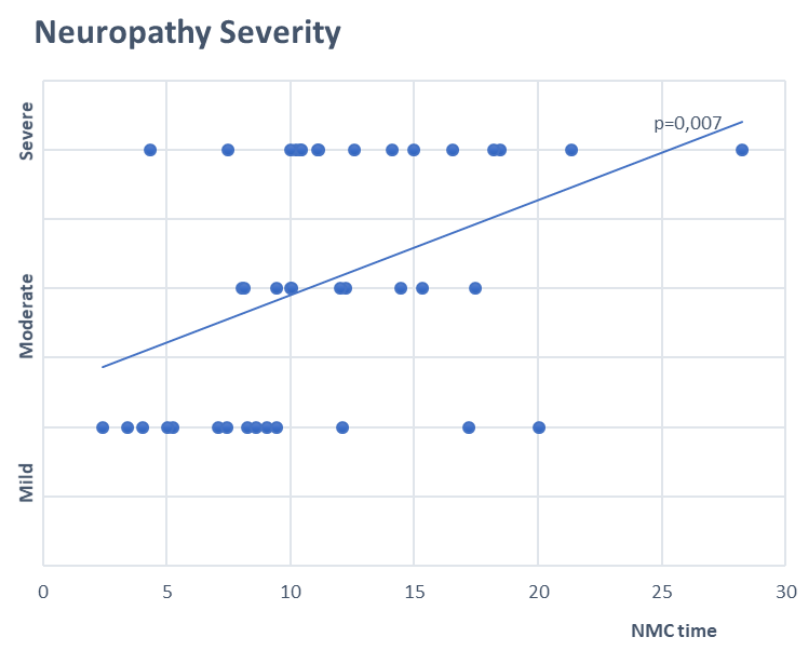

Figure 1: The NMC duration increased with the severity of polyneuropathy.

\section{Discussion}

Mucociliary clearance is one of the most important defense mechanisms of nasal respiratory epithelium. Harmful substances are retained in this mucous membrane and then removed from the nasal cavity by movements of cilia. Mucociliary interaction varies by environmental temperature, humidity, partial oxygen pressure, inhaled agents such as PO2, pH, trauma and sulfur dioxide, formaldehyde, ozone, chlorine, and smoking. It has been also reported that MC differs by age, hypertension, viral infections, chronic sinusitis, chronic and allergic rhinitis, anatomical obstacles such as adenoid hypertrophy, cystic fibrosis, chronic bronchitis, septum deviation, secondary to surgeries, bronchial asthma, multiple sclerosis, and DM (10-12).

There are many studies and case reports on susceptibility to DM and ENT diseases in the lit-erature. The most common presentations in the head and neck are otitis externa, hypoacusis, vertigo, dry mouth, dysphagia, fungal, and recurrent bacterial infections. However, changes in the nasal mucosa in DM are not well known. The main complaints of patients about the nose are xeromycteria, hyposmia, and reduced degrees of patency of the nose. Symptoms of chron-ic atrophic rhinitis, septal perforation, nasal mucosa ulcer, alar necrosis, staphylococcus or fungal infection may be seen in the otolaryngology examination (13-15). Yue compared mu-cociliary function in 50 insulin-dependent DM patients and 50 non-diabetic patients. He found that the mean value of nasal mucociliary clearance was significantly reduced in diabetic patients due to dry nose and increased $\mathrm{pH}$ values. He argued that the nasal mucociliary clear-ance is impaired as a result of increased viscosity due to electrolyte and water loss from the whole body and osmotic diuresis due to small vessel abnormalities (9).
There are consecutive studies in the literature confirming impaired nasal mucociliary clearance in diabetic patients (16-17).

In our study, the presence of polyneuropathy in DM patients was correlated with prolonged NMC duration. Polyneuropathy and autonomic nervous system involvement are one of the most common and most challenging complications of DM (18). DPN is the most common form of neuropathy worldwide and recent crosssectional studies from the US and Europe have reported a prevalence of diabetic peripheral neuropathy ranging from $6 \%$ to $51 \%$ de-pending on the population studied $(19,20)$. Although the presence of electrodiagnostic poly-neuropathy depends on multiple variables, chronic hyperglycemia significantly increases the risk and quantitatively reflect the severity of polyneuropathy in diabetic patients (21).

The most exciting finding of our study was that the severity of polyneuropathy and duration of NMC were significantly correlated. Dyck et al's classification of polyneuropathy is the most reliable classification used to measure the severity of DSPN in a given patient, to predict symptoms, symptoms, neurophysiological test results, and overall severity of DSPN, to con-duct medical practice and epidemiological studies and randomized controlled clinical trials (21). Although it can be attributed to multiple factors, autonomic control of the nasal mucosa should also be kept in mind. The autonomic nervous system richly innervates the nasal cavity and paranasal sinuses. Nonetheless, its contribution to sinonasal symptoms is poorly re-searched and understood (8).

The biggest limitation of our study was that no autonomic nervous system test, including sudomotor axon reflex test was performed in the routine of our electromyography laboratory. Assessment of sudomotor dysfunction would contribute to detection of autonomic dysfunc-tion in DPN. Although we could not specifically show autonomic dysfunction in our study, we speculate that it affects the nasal dryness that was emphasized in previous studies with DM patients. In our study, it was clearly seen that the severity of diabetic or non-diabetic pol-yneuropathy increased with the duration of NMC. In both of our neuropathy groups, there was a prolonged duration of NMC compared to the group of patients without neuropathy. In the DPN group, even longer duration of NMC was not statistically significant compared to the N-DPN group.

Our two most significant results were the presence of electrodiagnostic polyneuropathy, caus-ing more pronounced NMC dysfunction compared to DM patients without polyneuropathy, and increase in the severity of diabetic or non-diabetic polyneuropathy with the NMC dysfunction.

The fact that nasal mucociliary activity is negatively affected in diabetes mellitus is not a new phenomenon. However, according to our knowledge; this is the first and only study to show that the presence and degree of polyneuropathy in DM, in other words, the presence of prolonged hyperglycemia, affects NMC more prominently. 


\section{Conclusion}

In conclusion, patients with diabetes mellitus are a special group, and preventable problems should be taken into consideration when examining nasal pathologies. It should be kept in mind that NMC dysfunction can be both a cause and a result in the management of DM pa-tients, and thus it should be evaluated carefully.

Author Contributions: MAU: Project design, review of the literature, writing and revisions; SB: data collection and analyzes

Ethical issues: All authors declare originality and ethical approval of research. Responsibilities of research, responsibilities against local ethics commission are under the authors responsi-bilities. The study was conducted under defined rules by the local ethics commission guide-lines and audits.

Conflict of Interest: The authors declare that they have no competing interest.

\section{References}

1. Bustamante-Marin XM, Ostrowski LE. Cilia and Mucociliary Clearance. Cold Spring Harb Perspect Biol. 2017;9(4): a028241.

2. Houtmeyers SE, Gosselink R, Gayan-Ramirez G, Decramer M. Regulation of mucociliaray clearence in health and disease. Eur respir J 1999; 13:1177-1188

3. Xavier RF, Ramos D, Ito JT, Rodrigues FMM, Bertolini GN, Macchione M, et al. Effects of Cigarette Smoking Intensity on the Mucociliary Clearance of Active Smokers. Respiration 2013; $86: 479-485$

4. Jiao J, Zhang L. Influence of Intranasal Drugs on Human Nasal Mucociliary Clearance and Ciliary Beat Frequency. Allergy Asthma Immunol Res. 2019;11(3):306-319.

5. Corbo GM, Foresi A, Bonfitto P, Mugnano A, Agabiti N, Cole PJ. Measurement of nasal mucociliary clearance. Arch Dis Child. 1989;64(4):546-550.

6. Grossan M. Mucociliary Clearance: Measures and Therapies. Otolaryngol (Sunnyvale) 2017;7: 336

7. Rutland J, Cole PJ. Nasal mucociliary clearance and ciliary beat frequency in cystic fibrosis compared with sinusitis and bronchiectasis. Thorax. 1981;36(9):654-658

8. Yao A, Wilson JA, Ball SL. Autonomic nervous system dysfunction and sinonasal symptoms. Allergy Rhinol (Providence). 2018; $9 \cdot 2152656718764233$
9. Yue WL. Nasal mucociliary clearance in patients with diabetes mellitus. The Journal of Laryngology and Otology 1989; 103: 853 55 .

10. Proença de Oliveira-Maul J, Barbosa de Carvalho H, Goto DM Maia RM, Fló C, Barnabé V, et al. Aging, diabetes, and hypertension are associated with decreased nasal mucociliary clearance. Chest. 2013 Apr;143(4) 1091-1097.

11. Deniz M, UsluC, Ogredik EA, AkdumanD, Gursan SO. Nasa mucociliary clearance in total laryngectomized patients. Eur Arch Otorhinolaryngol 2006; 263: 1099-1104.

12. Sahin E, Hamamc1 M, Kantekin Y. Measurement of mucociliary clearance in the patients with multiple sclerosis. Eur Arch Otorhinolaryngol. 2020; 277(2) 469-473

13. Müller M, Betlejewski S. Nasal mucosa in patients with diabetes mellitus. Otolaryngol Pol. 2003; 57:361-364.

14. Zhang Z, Adappa ND, Lautenbach E, Chiu AG, Doghramji L, Howland TJ, et al. The effect of diabetes mellitus on chronic rhinosinusitis and sinus surgery outcome. Int Forum Allergy Rhinol. 2014 Apr;4(4):315-20.

15. Dinesh R, Avatar S, Haron A, Suhana MD, Azwarizan MD. Nasal septal abscess with uncontrolled diabetes mellitus: case reports. Med J Malaysia. $2011 ; 66(3): 253-4$.

16. Sachdeva A, Sachdeva OP, Gulati SP, Kakkar V. Nasal mucociliary clearance \& mucus $\mathrm{pH}$ in patients with diabetes mellitus. The Indian Journal of Medical Research. 1993; 98:265-268.

17. Selimoglu MA, Selimoglu E, Kurt A. Nasal mucociliary clearance and nasal and oral $\mathrm{pH}$ in patients with insulin-dependent diabetes. Ear, Nose, \& Throat Journal. 1999; 78:585-588.

18. Dyck PJ, Albers JW, Andersen H, Arezzo JC, Biessels GJ, Bril V, et al; Toronto Expert Panel on Diabetic Neuropathy. Toronto Expert Panel on Diabetic Neuropathy. Diabetic polyneuropathies: update on research definition, diagnostic criteria and estimation of severity. Diabetes Metab Res Rev. 2011; 27:620-628.

19. Iqbal Z, Azmi S, Yadav R, Ferdousi M, Kumar M, Cuthbertson DJ et al.Diabetic Peripheral Neuropathy: Epidemiology, Diagnosis, and Pharmacotherapy. Clin Ther. 2018; 40:828-849.

20. Hicks CW, Selvin E. Epidemiology of Peripheral Neuropathy and Lower Extremity Disease in Diabetes. Curr Diab Rep. 2019; 19: 86.

21. Lee WJ, Jang S, Lee SH, Lee HS. Correlation Between the Severity of Diabetic Peripheral Polyneuropathy and Glycosylated Hemoglobin Levels: A Quantitative Study. Ann Rehabil Med 2016:40(2):263-270. 